\title{
Three-Dimensional MHD Simulations of a Subcluster Plasma Moving in Turbulent ICM
}

\author{
N. Asai ${ }^{1}$, N. Fukuda ${ }^{2}$, and R. Matsumoto ${ }^{3}$ \\ ${ }^{1}$ Graduate School of Science and Technology, Chiba University, 1-33 Yayoi-cho, Inage-ku, \\ Chiba 263-8522, Japan \\ email: asai@astro.s.chiba-u.ac.jp \\ ${ }^{2}$ Department of Computer Simulation, Faculty of Informatics, Okayama University of Science, \\ 1-1 Ridai-cho, Okayama 700-0005, Japan \\ ${ }^{3}$ Department of Physics, Faculty of Science, Chiba University, 1-33 Yayoi-cho, Inage-ku, Chiba \\ 263-8522, Japan
}

\begin{abstract}
We carried out 3D magnetohydrodynamic simulations of a subcluster moving in turbulent ICM by including anisotropic heat conduction. Since magnetic fields stretched along the subcluster surface suppress the heat conduction across the front, cold fronts are formed and sustained.
\end{abstract}

Keywords. MHD, galaxies: interactions, galaxies: magnetic fields, X-rays: galaxies: clusters

Cold fronts observed by Chandra in merging clusters are sharp discontinuities of X-ray surface brightness and temperature. Their existence is puzzling unless the heat conductivity across the front reduced enough. By extending our previous 2D magnetohydrodynamic (MHD) simulation including anisotropic heat conduction (Asai, Fukuda \& Matsumoto 2004), we carried out 3D MHD simulations of a subcluster moving in magnetically turbulent ICM. Figure. 1 shows numerical results. The cold front formed in a leading edge of the subcluster is maintained for 1 Gyr because the subcluster is almost entirely covered with stretched magnetic fields, which sufficiently suppress the heat conduction across the front. We conclude that magnetic fields play an essential role for the coexistence of hot and cold plasma in clusters of galaxies.
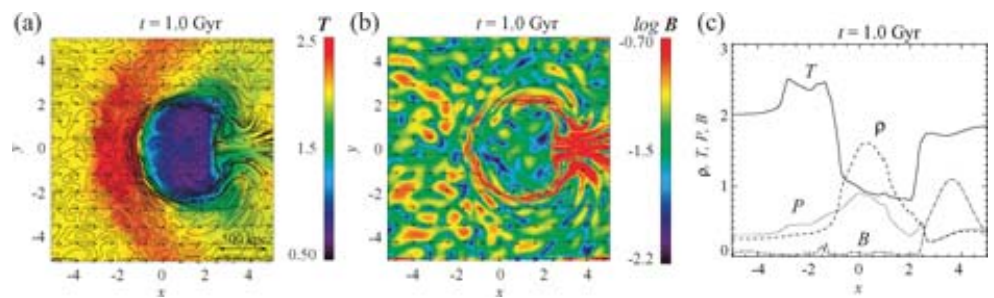

Figure 1. Distributions of (a) temperature and (b) magnetic field strength along $z=0$ plane at $t=1.0$ Gyr. Contours and arrows show magnetic field lines and velocity vectors. (c) Distributions of temperature (solid line), density (dashed line), pressure (dotted line), and magnetic field strength (dot-dashed line) along $x$-axis at $t=1.0 \mathrm{Gyr}$, respectively.

This work is supported by JSPS Research Fellowships for Young Scientists. Numerical computations are carried out by VPP5000 at NAOJ and joint research program of IMIT, Chiba University.

\section{Reference}

Asai, N., Fukuda, N. \& Matsumoto, R. 2004, ApJ (Letters) 606, L105. 